\title{
RapidArc, intensity modulated photon and proton techniques for recurrent prostate cancer in previously irradiated patients: a treatment planning comparison study
}

\author{
Damien C Weber*1,5, Hui Wang1, Luca Cozzi², Giovanna Dipasquale ${ }^{1}$, \\ Haleem G Khan ${ }^{3}$, Osman Ratib ${ }^{4,5}$, Michel Rouzaud ${ }^{1}$, Hansjoerg Vees ${ }^{1}$, \\ Habib Zaidi ${ }^{4}$ and Raymond Miralbell ${ }^{1,5}$
}

\begin{abstract}
Address: ${ }^{1}$ Department of Radiation Oncology, University Hospital of Geneva, Geneva, Switzerland, ${ }^{2}$ Oncology Institute of Southern Switzerland, Medical Physics Unit, Bellinzona, Switzerland, ${ }^{3}$ Institute of Radiology Jean Violette, Geneva, Switzerland, ${ }^{4}$ Department of Nuclear Medicine, University Hospital of Geneva, Geneva, Switzerland and ${ }^{5}$ Faculty of medicine, UNIGE, University of Geneva, Switzerland

Email: Damien C Weber* - damien.weber@hcuge.ch; Hui Wang - hui.wang@hcuge.ch; Luca Cozzi - luca.cozzi@iosi.ch; Giovanna Dipasquale - giovanna.dipasquale@hcuge.ch; Haleem G Khan - khanhaleem@hotmail.com; Osman

Ratib - ratib-osman@diogenes.hcuge.ch; Michel Rouzaud - michel.rouzaud@hcuge.ch; Hansjoerg Vees - hansjoerg.vees@hcuge.ch; Habib Zaidi - habib.zaidi@hcuge.ch; Raymond Miralbell - raymond.miralbell@hcuge.ch

* Corresponding author
\end{abstract}

Published: 9 September 2009

Radiation Oncology 2009, 4:34 doi:10.1186/1748-717X-4-34

This article is available from: http://www.ro-journal.com/content/4/I/34

(C) 2009 Weber et al; licensee BioMed Central Ltd.

This is an Open Access article distributed under the terms of the Creative Commons Attribution License (http://creativecommons.org/licenses/by/2.0), which permits unrestricted use, distribution, and reproduction in any medium, provided the original work is properly cited.
Received: 2 June 2009

Accepted: 9 September 2009

\begin{abstract}
Background: A study was performed comparing volumetric modulated arcs (RA) and intensity modulation (with photons, IMRT, or protons, IMPT) radiation therapy (RT) for patients with recurrent prostate cancer after RT.

Methods: Plans for RA, IMRT and IMPT were optimized for 7 patients. Prescribed dose was 56 $\mathrm{Gy}$ in 14 fractions. The recurrent gross tumor volume (GTV) was defined on ${ }^{18} \mathrm{~F}$-fluorocholine PET/ CT scans. Plans aimed to cover at least $95 \%$ of the planning target volume with a dose $>50.4 \mathrm{~Gy}$. A maximum dose $\left(D_{\text {Max }}\right)$ of 61.6 Gy was allowed to $5 \%$ of the GTV. For the urethra, $D_{\text {Max }}$ was constrained to $37 \mathrm{~Gy}$. Rectal $\mathrm{D}_{\text {Median }}$ was $<17 \mathrm{~Gy}$. Results were analyzed using Dose-Volume Histogram and conformity index $\left(\mathrm{Cl}_{90}\right)$ parameters.

Results: Tumor coverage (GTV and PTV) was improved with RA ( $\mathrm{V}_{95 \%} 92.6 \pm 7.9$ and $\left.83.7 \pm 3.3 \%\right)$, when compared to IMRT $\left(\mathrm{V}_{95 \%} 88.6 \pm 10.8\right.$ and $\left.77.2 \pm 2.2 \%\right)$. The corresponding values for IMPT were intermediate for the GTV $\left(V_{95 \%} 88.9 \pm 10.5 \%\right)$ and better for the PTV $\left(\mathrm{V}_{95 \%} 85.6 \pm 5.0 \%\right)$. The percentages of rectal and urethral volumes receiving intermediate doses (35 Gy) were significantly decreased with RA $(5.1 \pm 3.0$ and $38.0 \pm 25.3 \%)$ and IMPT $(3.9 \pm 2.7$ and $25.1 \pm 21.1 \%)$, when compared to IMRT $(9.8 \pm 5.3$ and $60.7 \pm 41.7 \%)$. $\mathrm{Cl}_{90}$ was $\mathrm{I} .3 \pm 0.1$ for photons and I.6 \pm 0.2 for protons. Integral Dose was I.I $\pm 0.5 \mathrm{~Gy}^{*} \mathrm{~cm}^{3} * 10^{5}$ for IMPT and about a factor three higher for all photon's techniques.
\end{abstract}

Conclusion: RA and IMPT showed improvements in conformal avoidance relative to fixed beam IMRT for 7 patients with recurrent prostate cancer. IMPT showed further sparing of organs at risk. 


\section{Background}

Biochemical failures (BF) of prostate cancer after external beam radiation therapy (RT) is not an unusual event and is observed in a substantial number of prostate cancer patients [1,2]. CapSURE ${ }^{\mathrm{TM}}$ (Cancer of the Prostate Strategic Urologic Research Endeavor) data have demonstrated a biochemical failure rate following radiation therapy as high as $63 \%$ [3]. Up to $70 \%$ of these patients will have evidence of recurrent or residual disease within the prostate gland [4]. Although curative treatment is still an option if the patient presents organ-confined disease only, no consensus exists however on the optimal salvage therapy modality for these patients. Therapeutic management of these patients includes salvage radical prostatectomy, cryotherapy, brachytherapy or high-intensity focused ultrasound, with or without hormonal deprivation therapy. Re-irradiation with conformal techniques is yet another strategy with potential curative intent. Re-irradiation techniques must however minimally deliver radiation dose to pre-irradiated organ at risk (OARs) in the direct vicinity of the target volume.

The demonstration of organ-confined only recurrent disease in patients with BF is not easily done with conventional radiology. Identifying precisely the target recurrent volume is of paramount importance when delivering focused high-radiation dose in a pre-irradiated area. Recent progress in imaging with PET tracers such as acetate or choline labelled with ${ }^{11} \mathrm{C}$ or ${ }^{18} \mathrm{~F}$ have improved significantly the accuracy in diagnosing the site of relapse [5]. Local tracer uptake within the gland may correspond to the locally recurring gross-tumor volume (GTV) and can be contoured in the RT treatment planning system.

RapidArc (RA), is a novel technique which may achieve several objectives: i) improve organ at risks (OARs) and non-target tissue sparing compared to other intensity modulated RT (IMRT) techniques; ii) maintain or improve the same degree of target coverage; iii) reduce significantly the treatment time per fraction. Dose comparative studies using RA, have been published in prostate $[6,7]$, cervix uteri [8] and anal canal cancer [9], showing significant improvements when compared to non-RA techniques. This technique could be thus used to treat geometrically complex partial recurrent tumor volumes within the prostate gland after RT.

The present study was undertaken to assess the treatment planning inter-comparison between photon and proton RT, namely IMRT and IMPT, to RA, as applied to a total of 7 recurrent pre-irradiated prostate cancer patients

\section{Methods}

The institutional ${ }^{18} \mathrm{~F}$-Choline database containing 47 prostate cancer patients was queried to identify individuals with: 1) biochemically recurrence; 2) local relapse only; 3 ) previous high-dose ( $\geq 70 \mathrm{~Gy}$ ) RT and 4) endorectal MRI. Seven of such patients were identified (median age, 77 years; Table 1). They all underwent previous curative 3D conformal RT (median dose, 74 Gy; HDR brachytherapy boost 14 Gy in 2 fractions, 2 patients), 4.8 to 7.6 (median, 5.9) years before biological recurrence (Table 1).

Table I: Patients characteristics

\begin{tabular}{|c|c|c|c|c|c|c|c|}
\hline No of patient & $\mathbf{I}$ & 2 & 3 & 4 & 5 & 6 & 7 \\
\hline Age (years) & 81 & 63 & 79 & 69 & 77 & 78 & 69 \\
\hline Recurrence time (years) & 5.86 & 4.82 & 6.75 & 5.16 & 5.85 & 5.82 & 7.55 \\
\hline PSADT (month) & 13.9 & 9.0 & 10.2 & 8.5 & 10.2 & 5.5 & 25.8 \\
\hline Tumour stage (at relapse) & $\mathrm{T} 2 \mathrm{c}$ & $\mathrm{T} 3 \mathrm{~b}$ & $\mathrm{~T} 2 \mathrm{c}$ & $\mathrm{T} 2 \mathrm{c}$ & T3a & $\mathrm{T} 2 \mathrm{c}$ & T3b \\
\hline PSA at recurrence $(\mathrm{ng} / \mathrm{ml})$ & 5.11 & 6.76 & 2.80 & 5.14 & 6.32 & 5.95 & 13.00 \\
\hline Gleason score at recurrence & $3+4$ & - & $3+4$ & $3+3$ & $4+3$ & $4+3$ & $3+3$ \\
\hline $\operatorname{GTV}\left(\mathrm{cm}^{3}\right)$ & 0.61 & 1.09 & 3.48 & 5.08 & 5.75 & 10.36 & 19.93 \\
\hline $\operatorname{CTV}\left(\mathrm{cm}^{3}\right)$ & 2.59 & 3.29 & 9.72 & 12.84 & 15.65 & 20.91 & 38.61 \\
\hline $\operatorname{PTV}\left(\mathrm{cm}^{3}\right)$ & 6.68 & 8.13 & 22.13 & 26.67 & 30.42 & 39.47 & 64.20 \\
\hline
\end{tabular}

Abbreviations: PSA = prostate-specific antigen; PSADT = prostate-specific antigen doubling time; PET-CT = Positron Emission Tomography and Computed Tomography; GTV = gross tumour volume; CTV = Clinical Target Volume; PTV = Planning Target Volume. 
The median dose received by $50 \% / 1 \%$ of the rectum and bladder by this prior treatment were 44.1 (range, 60.0 38.5)/71.0 (range, 74.5 - 62.4) and 59.0 (range, 67.2 43.4)/74.0 (range, 78.0 - 64.4) Gy, respectively. The median rectal volume receiving 35 Gy was $79.4 \%$, and range from 56.0 to $96.0 \%$. Local relapse was proven by PET-CT examination with ${ }^{18} \mathrm{~F}$-choline; failures were confirmed by sextant biopsy in all but one patient. A positive correlation between ${ }^{18} \mathrm{~F}$-choline uptake and the location of the histological proven recurrence was observed in all 6 patients. Table 2 details the radiological and pathological correlation of these recurrences. PET/CT imaging was performed on the Biograph 16 scanner (Siemens Medical Solution, Erlangen, Germany) operating in 3D mode (Fig. 1). An endorectal MRI, with spectroscopy and contrast enhancement, was acquired for all patients [10]. The main organs at risk (OARs) considered for all patients were the urethra (defined on the base of MR imaging and verified by an experienced radiologist), bladder, rectum, penile bulb and femoral heads The non-target tissue was defined as the patient's volume covered by the CT scan minus the planning target volume (PTV).

For all patients, GTV was outlined using the signal-tobackground ratio-based adaptive thresholding technique described in [11] and adapted to our PET/CT scanner characteristics. Data acquisition and processing protocols are described elsewhere [12]. The clinical applicability of detecting prostate recurrence with $18 \mathrm{~F}$-Choline PET has been demonstrated in our previous series [13]. Fig. 1 depicts the PET GTV for 1 patient. Clinical target volume (CTV) was defined adding a 3D anisotropic margin of 3 $\mathrm{mm}$ (CTV was however limited to the prostate and seminal vesicles and could not be stretched beyond these struc- tures), excluding the urethra in all cases. PTV was defined adding a 3D anisotropic margin of $3 \mathrm{~mm}(2 \mathrm{~mm}$ in proximity of the urethra) to the CTV. A summary of the sizes of the GTVs, PTVs and OARs are detailed in Tables 1 and 2.

Dose prescription of 56 Gy to PTV was delivered according to a hypofractionated radiation schedule consisting of 14 daily fractions of $4 \mathrm{~Gy}$, twice weekly (overall treatment time, 7 weeks) [14]. All plans were normalized to the mean dose of the PTV.

Plans aimed to cover at least $95 \%$ of the PTV with a dose greater than $90 \%$ of the dose prescription. An over-dosage of maximum 61.6 Gy (110\%) was allowed to 5\% of both CTV and PTV. For the urethra, the maximum dose was constrained to $37 \mathrm{~Gy}$. A dose lower than 28 Gy delivered to $50 \%$ of the volume of the bladder, penile bulb and femoral heads was required for these OARs; likewise, a dose < 17 Gy was constraint to $30 \%$ of the rectal volume.

Four sets of plans were compared in this study, all designed on the Varian Eclipse treatment planning system (version 8.6.10) with $6 \mathrm{MV}$ photon beams from a Varian Clinac equipped with either a Millennium Multileaf Collimator (MLC) with 120 leaves (RA_M120; spatial resolution of $5 \mathrm{~mm}$ at isocentre) or a High Definition MLC with 120 leaves (RA_HD120; spatial resolution of $2.5 \mathrm{~mm}$ at isocentre). Plans for RA were optimized selecting a maximum DR of $600 \mathrm{MU} / \mathrm{min}$ and a fixed DR of $600 \mathrm{MU} / \mathrm{min}$ was selected for IMRT.

The Anisotropic Analytical Algorithm photon dose calculation algorithm was used for all photon cases [15]. The dose calculation grid was set to $2.5 \mathrm{~mm}$.

Table 2: Prostate cancer recurrence on MRI, PET and biopsy

\begin{tabular}{cccc}
\hline & \multicolumn{3}{c}{ Recurrent Site } \\
\cline { 2 - 4 } No of patient & MRI & PET CT & Biopsy (Number of positive cores) \\
\hline I & L, R & L & L (I/7); R (0/6) \\
\hline 3 & SV & SV & L (I/3); R (3/4) \\
\hline 4 & L, R & L, R & L (0/4); R (3/4) \\
\hline 5 & R & R & L (2/3); R (I/3) \\
\hline 6 & L & L, R & L (3/3); R (4/4)
\end{tabular}

Abbreviations: L, left prostate lobe; R, right prostate lobe; SV, seminal vesicle; ND, not done. 
A

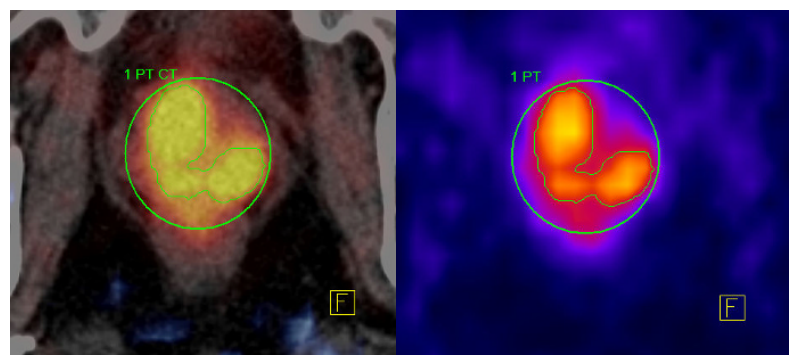

B

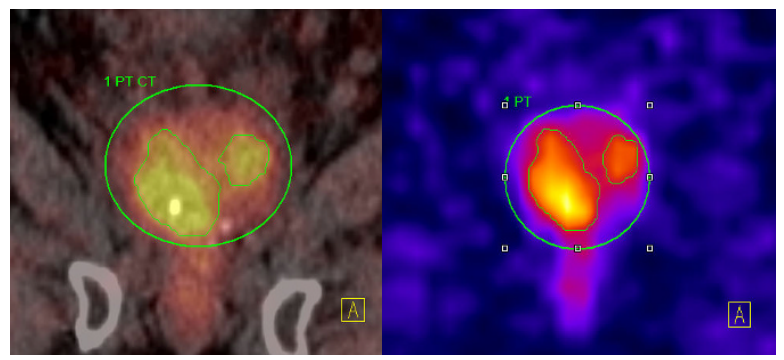

C

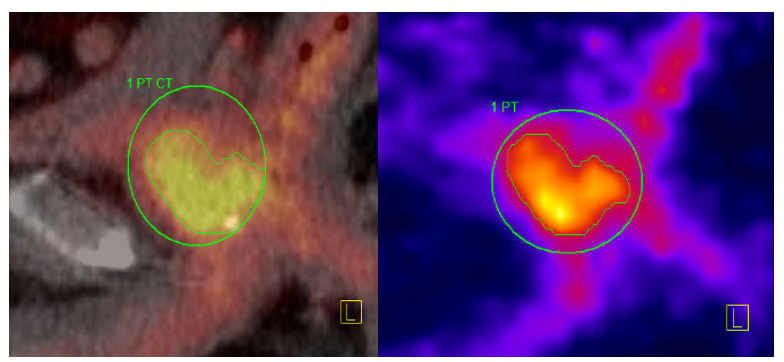

Figure I

GTV in the axial (A), coronal (B) and sagital (C) simulation CT with PET fusion and I8F-choline PET slice, respectively.

\section{RA}

RA uses continuous variation of the instantaneous dose rate, MLC leaf positions and gantry rotational speed to optimize the dose distribution. Details about RA optimization process have been published elsewhere [8]. To minimize the contribution of tongue and groove effect during the arc rotation and to benefit from leaves trajectories non-coplanar with respect to patient's axis, the collimator rotation in RA remains fixed to a value different from zero. In the present study collimator was rotated to $\sim 30^{\circ}$ depending on the patient.

For the study, two sets of plans were optimized, each with a single $\operatorname{arc} 360^{\circ}$. The first set (RA_M120) was created using the Millennium MLC, the second set (RA_HD120) with the High Definition MLC.

\section{IMRT}

Plans were designed according to the dynamic sliding window method [16] with five fixed gantry beams. One single isocentre was located at the target center of mass. All beams were coplanar with collimator angle set to $0^{\circ}$. The Millennium MLC was used for the study.

\section{IMPT}

Intensity modulated proton plans were obtained for a generic proton beam through a spot scanning optimization technique implemented in the Eclipse treatment planning system from Varian. The optimization process has been detailed elsewhere [17]. Spot spacing was set to $3 \mathrm{~mm}$, circular lateral target margins were set to $5 \mathrm{~mm}$, proximal margin to $5 \mathrm{~mm}$ and distal margin to $2 \mathrm{~mm}$. In all cases coplanar beam arrangement was adopted using 3 fields, one with posterior and two with anterior oblique incidence.

Quantitative evaluation of plans was performed by means of standard Dose-Volume Histogram (DVH). For GTV and PTV, the values of $\mathrm{D}_{98 \%}$ and $\mathrm{D}_{2 \%}$ (dose received by the $98 \%$ and $2 \%$ of the volume) were defined as metrics for minimum and maximum doses and thereafter reported. To complement the appraisal of minimum and maximum dose, $\mathrm{V}_{95 \%}$ and $\mathrm{V}_{107 \%}$ (the volume receiving at least $95 \%$ or at most $107 \%$ of the prescribed dose) were reported. The homogeneity of the treatment was expressed in terms of the standard deviation and of $\mathrm{D}_{5 \%}-\mathrm{D}_{95 \%}$. The conformality of the plans was measured with a Conformity Index, $\mathrm{CI}_{90 \%}$ defined as the ratio between the patient volume receiving at least $90 \%$ of the prescribed dose and the volume of the PTV.

For OARs, the analysis included the mean dose, the maximum dose expressed as $\mathrm{D}_{1 \%}$ and a set of appropriate volume $\left(V_{X}\right)$ and dose $\left(D_{Y}\right)$ metrics.

For non-target tissue, the integral dose, (Dose Int $_{\text {, }}$ ) is defined as the integral of the absorbed dose extended to over all voxels excluding those within the target volume (Dose $_{\text {Int }}$ dimensions are $\mathrm{Gy}^{*} \mathrm{~cm}^{3}$ ). This was reported together with the observed mean dose and some representative $\mathrm{V}_{\mathrm{x}}$ values.

To visualize the global difference between techniques, average cumulative DVH for GTV and PTV, OARs and healthy tissue, were built from the individual DVHs. These DVHs were obtained by averaging the corresponding volumes over the whole patient's cohort for each dose bin of $0.05 \mathrm{~Gy}$.

To appraise the difference between the techniques, the paired, two-tails Student's $t$-test was applied whenever applicable. Data were considered statistically significant for $\mathrm{p}<0.05$. 


\section{Results}

The mean prostate volume was $35.4 \pm 7.8 \mathrm{~cm}^{3}$ and the average GTV and PTV volumes are reported in Table 3. The mean ratio between PTV and prostate volume was $0.77 \pm$ 0.50 with a range from 0.19 to 1.76 .

For the GTV and PTV, the RA_HD120 and IMRT techniques produced the best and worst dose homogeneity, respectively (Table 3 ). The GTV coverage was optimal with RA (mean $\mathrm{V}_{95 \%} 92 \%$; Table 3 ). The PTV coverage $\left(\mathrm{V}_{95 \%}\right)$ was better with IMPT, intermediate with RA and worse with IMRT (Table 3).

The GTV and PTV $V_{95 \%}$-difference observed between RA_HD120 and RA_M120 (Table 3) is due to different MLC characteristics, namely spatial resolution and transmission. IMPT showed a moderate improvement compared to IMRT ( $\mathrm{V}_{107}$ and $\mathrm{V}_{95}$; Table 3 ). Interestingly, IMPT did not reach the performance of RA_HD120 for $\mathrm{V}_{107}$ for both the GTV and PTV (Table 3). None of the techniques achieved the planning objective on minimum PTV dose
(Table 3). IMRT failed to reach the objective on $\mathrm{D}_{5 \%}$ for PTV while all others met the condition (Table 3 ).

The rectal dose was significantly decreased with IMPT and RA, respectively (Fig. 2, 3). For the intermediate dose level, these two techniques more than halved the percentage of rectal volume receiving 35 and $45 \mathrm{~Gy}$ (Table 4). For the high-dose level, IMPT delivered a decreased dose when compared to the other two photons techniques (Table 4).

For the urethra, none of the techniques was able to keep the maximum dose below the threshold of 37 Gy (Table 4). IMPT violated this dose level by approximately $1 \mathrm{~Gy}$, while RA and IMRT exceeded this metric by 2.3 - 2.8 and 3 Gy, respectively. For the intermediate dose level, IMPT and RA approximately halved the percentage of urethral volume receiving 35 and 45 Gy (Table 4), respectively. Since the urethra was included in the PTV in a majority (5/ 7 ) of patients, the observed values were expected.

Table 3: Dosimetric results for GTV and PTV

\begin{tabular}{|c|c|c|c|c|c|}
\hline Parameter & IMRT & IMPT & RA_HDI 20 & RA_MI 20 & $p$ \\
\hline \multicolumn{6}{|c|}{ GTV Volume $[\mathrm{cm} 3] 6.7 \pm 6.8[0.6-19.9]$} \\
\hline Mean [Gy] & $58.9 \pm 2.2$ & $56.5 \pm 1.0$ & $57.2 \pm 0.6$ & $57.3 \pm 0.8$ & $\mathrm{e}$ \\
\hline$D_{5}-D_{95}[G y]$ & $12.4 \pm 6.9$ & $12.5 \pm 6.0$ & $8.5 \pm 5.3$ & $10.2 \pm 5.3$ & a,b,c,d,e,f \\
\hline$D_{2}[G y]$ & $64.6 \pm 1.2$ & $61.9 \pm 2.7$ & $60.7 \pm 2.0$ & $61.5 \pm 1.6$ & $a, b, c, d$ \\
\hline$D_{98}[G y]$ & $49.3 \pm 7.7$ & $46.6 \pm 6.9$ & $49.2 \pm 6.6$ & $48.2 \pm 6.3$ & $d, e, f$ \\
\hline $\mathrm{V}_{95}[\%]$ & $88.6 \pm 10.8$ & $88.9 \pm 10.5$ & $92.6 \pm 7.9$ & $91.4 \pm 8.5$ & $d, e, f$ \\
\hline$V_{107}[\%]$ & $52.3 \pm 27.8$ & $21.1 \pm 14.9$ & $9.1 \pm 12.1$ & $19.3 \pm 14.2$ & $b, f$ \\
\hline \multicolumn{6}{|c|}{ PTV Volume $[\mathrm{cm} 3] 27.7 \pm 19.6[6.7-64.2]$} \\
\hline Mean [Gy] & $56.0 \pm 0.0$ & $56.0 \pm 0.0$ & $56.0 \pm 0.0$ & $56.0 \pm 0.0$ & \\
\hline$D_{5}-D_{95}[G y]$ & $15.0 \pm 2.0$ & $13.6 \pm 4.3$ & $11.8 \pm 2.7$ & $13.2 \pm 3.2$ & $a, b, c, d, f$ \\
\hline$D_{2}[G y]$ & $63.6 \pm 0.9$ & $61.4 \pm 1.6$ & $60.7 \pm 1.5$ & $61.5 \pm 1.3$ & $a, b, c, d, f$ \\
\hline$D_{5}[G y]$ & $62.3 \pm 0.9$ & $60.7 \pm 1.4$ & $60.0 \pm 1.2$ & $60.7 \pm 1.2$ & $a, b, c, d, f$ \\
\hline $\mathrm{D}_{98}[\mathrm{~Gy}]$ & $43.8 \pm 2.8$ & $42.4 \pm 5.4$ & $44.1 \pm 4.0$ & $43.5 \pm 4.5$ & $d, e, f$ \\
\hline $\mathrm{V}_{95}[\%]$ & $77.2 \pm 2.2$ & $85.6 \pm 5.0$ & $83.7 \pm 3.3$ & $81.8 \pm 4.2$ & a,b,e,f \\
\hline$V_{107}[\%]$ & $18.2 \pm 2.6$ & $12.6 \pm 8.5$ & $6.9 \pm 6.4$ & $12.5 \pm 8.6$ & $b, d, f$ \\
\hline
\end{tabular}

$a=I M R T$ vs IMPT b = IMRT vs RA_HDI $20 \mathrm{c}=$ IMRT vs RA_MI 20

$\mathrm{d}=$ IMPT vs RA_HDI $20 \mathrm{e}=$ IMPT vs RA_MI $20 \mathrm{f}=$ RA_HDI 20 vs RA_MI 20 
Table 4: Dosimetric results for OARs and non target tissues

\begin{tabular}{|c|c|c|c|c|c|}
\hline Parameter & IMRT & IMPT & RA_HDI 20 & RA_MI20 & $p$ \\
\hline \multicolumn{6}{|c|}{ Rectum. Volume $[\mathrm{cm} 3] 48.6 \pm$ I $7.6[28.4-72.5]$} \\
\hline$D_{50}[G y]$ & $10.1 \pm 6.2$ & $4.1 \pm 4.0$ & $8.2 \pm 3.9$ & $9.1 \pm 4.2$ & $a, b, d, e, f$ \\
\hline$D_{1}[G y]$ & $49.6 \pm 6.8$ & $45.1 \pm 9.2$ & $45.2 \pm 8.3$ & $46.5 \pm 7.8$ & $a, b, c$ \\
\hline $\mathrm{V}_{35 \mathrm{~Gy}}[\%]$ & $9.8 \pm 5.3$ & $3.9 \pm 2.7$ & $5.1 \pm 3.0$ & $5.9 \pm 3.3$ & $a, b, c, e$ \\
\hline $\mathrm{V}_{45 \mathrm{~Gy}}[\%]$ & $3.6 \pm 2.4$ & $1.6 \pm 1.3$ & $1.6 \pm 1.1$ & $1.9 \pm 1.3$ & $a, b, c$ \\
\hline \multicolumn{6}{|c|}{ Urethra. Volume $[\mathrm{cm} 3] 0.7 \pm 0.1[0.6-0.8]$} \\
\hline $\mathrm{D}_{50}[\mathrm{~Gy}]$ & $31.4 \pm 13.1$ & $26.8 \pm 11.7$ & $28.6 \pm 11.4$ & $28.6 \pm 10.9$ & $\mathrm{a}, \mathrm{b}, \mathrm{c}, \mathrm{d}, \mathrm{e}$ \\
\hline$D_{1}[G y]$ & $40.1 \pm 3.3$ & $38.1 \pm 2.4$ & $39.8 \pm 3.5$ & $39.3 \pm 3.3$ & $a, c, d, f$ \\
\hline $\mathrm{V}_{35 \mathrm{~Gy}}[\%]$ & $60.7 \pm 41.7$ & $25.1 \pm 21.1$ & $38.0 \pm 25.3$ & $36.0 \pm 24.0$ & $a, b, c$ \\
\hline $\mathrm{V}_{40 \mathrm{~Gy}}[\%]$ & $11.0 \pm 12.8$ & $0.6 \pm 1.1$ & $5.1 \pm 5.4$ & $4.0 \pm 5.6$ & \\
\hline \multicolumn{6}{|c|}{ Left femoral head Volume $[\mathrm{cm} 3] 60.1 \pm 4.4[54.8-67.6]$} \\
\hline $\mathrm{D}_{50}[\mathrm{~Gy}]$ & $3.9 \pm 2.6$ & $0.1 \pm 0.1$ & $3.3 \pm 2.1$ & $3.5 \pm 2.1$ & $a, b, d, e, f$ \\
\hline$\left.D_{1} G y\right]$ & $14.6 \pm 7.2$ & $2.3 \pm 2.0$ & $7.4 \pm 1.5$ & $7.6 \pm 1.3$ & $a, b, c, d, e$ \\
\hline \multicolumn{6}{|c|}{ Right femoral head Volume $[\mathrm{cm} 3] 60.9 \pm 5.8[54.6-71.6]$} \\
\hline $\mathrm{D}_{50}[\mathrm{~Gy}]$ & $3.9 \pm 2.7$ & $0.1 \pm 0.1$ & $3.2 \pm 2.3$ & $3.4 \pm 2.1$ & $\mathrm{a}, \mathrm{d}, \mathrm{e}$ \\
\hline$\left.D_{1} G y\right]$ & $15.3 \pm 7.5$ & $2.5 \pm 3.0$ & $8.0 \pm 1.8$ & $8.0 \pm 1.7$ & $\mathrm{a}, \mathrm{b}, \mathrm{c}, \mathrm{d}, \mathrm{e}$ \\
\hline \multicolumn{6}{|c|}{ Bladder. Volume $[\mathrm{cm} 3] 109.8 \pm 63.6[32.7-234.2]$} \\
\hline$D_{50}[G y]$ & $4.9 \pm 3.2$ & $0.7 \pm 0.9$ & $4.6 \pm 2.6$ & $5.2 \pm 3.0$ & $a, d, e, f$ \\
\hline$D_{1}[G y]$ & $42.3 \pm 17.0$ & $38.8 \pm 19.6$ & $41.3 \pm 16.3$ & $42.1 \pm 15.8$ & \\
\hline $\mathrm{V}_{35 \mathrm{~Gy}}[\%]$ & $6.4 \pm 6.3$ & $3.9 \pm 4.3$ & $4.1 \pm 4.1$ & $4.5 \pm 4.2$ & $\mathrm{a}$ \\
\hline $\mathrm{V}_{50 \mathrm{~Gy}}[\%]$ & $1.9 \pm 2.7$ & $1.4 \pm 2.1$ & $1.3 \pm 2.1$ & $1.3 \pm 2.1$ & \\
\hline \multicolumn{6}{|c|}{ Penile bulb. Volume $[\mathrm{cm} 3] 7.2 \pm 3.2[3.0-13.2]$} \\
\hline$D_{50}[G y]$ & $2.0 \pm 1.5$ & $0.9 \pm 1.4$ & $2.5 \pm 1.7$ & $3.2 \pm 2.5$ & a,b,c,d,e \\
\hline$D_{1}[G y]$ & $7.6 \pm 9.4$ & $7.1 \pm 9.0$ & $5.8 \pm 4.6$ & $7.7 \pm 7.4$ & \\
\hline \multicolumn{6}{|c|}{ Non Target Tissue } \\
\hline Mean $[G y]$ & $2.0 \pm 0.8$ & $0.7 \pm 0.3$ & $1.8 \pm 0.7$ & $1.9 \pm 0.7$ & a,b,d,e,f \\
\hline$V_{10 G y}[\%]$ & $6.0 \pm 2.6$ & $2.8 \pm 1.3$ & $4.7 \pm 2.5$ & $5.1 \pm 2.8$ & a,b,c,d,e \\
\hline $\mathrm{Cl}_{90}$ & $1.3 \pm 0.1$ & $1.6 \pm 0.2$ & $1.3 \pm 0.1$ & $1.3 \pm 0.1$ & $\mathrm{a}, \mathrm{d}, \mathrm{e}$ \\
\hline $\operatorname{Dose}_{\text {Int }}\left[\mathrm{Gy} * \mathrm{~cm}^{3} \mathrm{I}^{4}\right]$ & $3.3 \pm 1.6$ & $1.1 \pm 0.5$ & $2.9 \pm 1.3$ & $3.1 \pm 1.4$ & a,b,d,e,f \\
\hline
\end{tabular}

$\mathrm{a}=$ IMRT vs IMPT $\mathrm{b}=$ IMRT vs RA_HDI $20 \mathrm{c}=$ IMRT vs RA_MI 20

$\mathrm{d}=$ IMPT vs RA_HDI $20 \mathrm{e}=$ IMPT vs RA_MI $20 \mathrm{f}=$ RA_HDI 20 vs RA_MI 20 
IMPT resulted in an almost complete avoidance of femoral heads (Fig. 2; median inferior to $0.1 \mathrm{~Gy}$; Table 4) while both RA reduced maximum dose of about $50 \%$ compared to IMRT.

IMPT was the best technique to spare the penile bulb (Fig. $3)$. For the bladder, all non-IMPT techniques were identical (Table 4; Fig 3).

Non target tissue irradiation was limited for all techniques and the mean dose was kept under the Gy unit for the majority of patients (Table 4). IMPT showed a Dose Int $_{\text {of }}$ approximately a factor 3 lower than all the photon techniques. The $\mathrm{CI}$ was however better with photons techniques (mean CI improvement: 18\%), because of the wider lateral and distal spread induced by spot size, spacing and margins used to achieve sufficient target coverage (Table 4).

For all but one OARs (urethra), RA_HD120 results were better than those observed with RA_M120 (Table 4). This

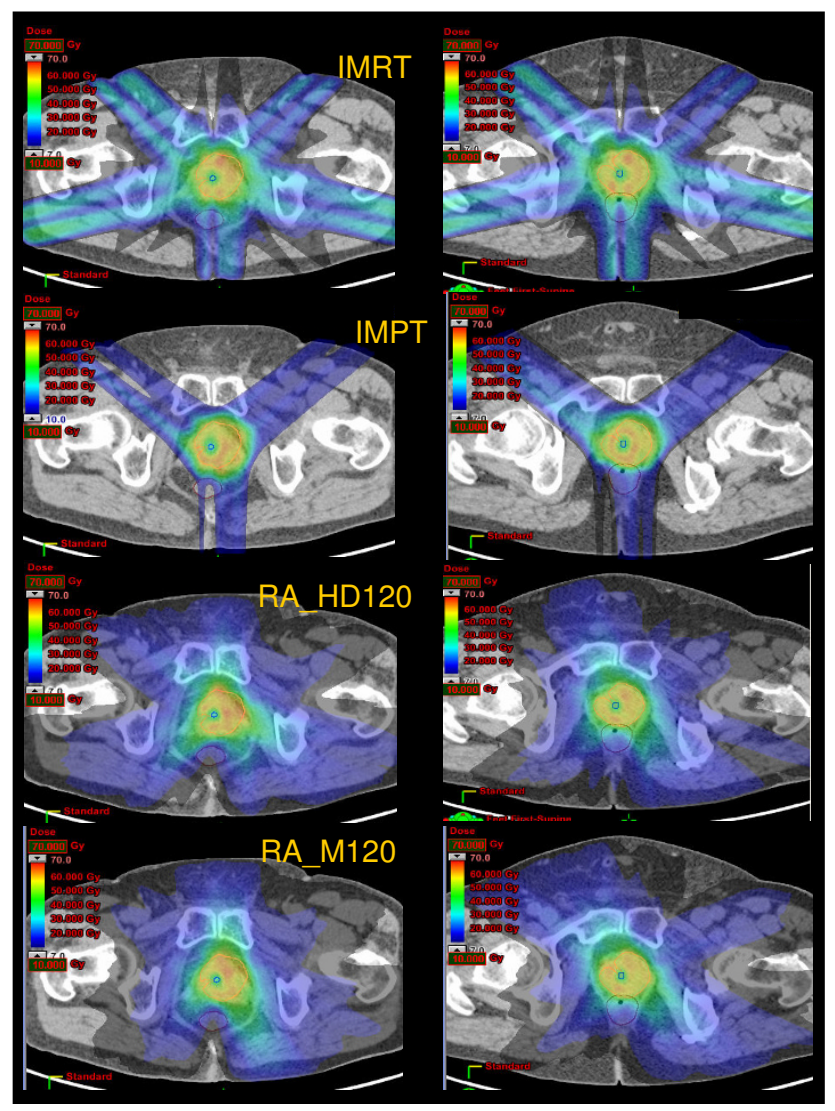

Figure 2

Color wash IMRT, IMPT, RA_HD I 20 and RA_MI 20 dose distributions for the planning target volume (PTV) for two patients with recurrent prostate cancer. observed OAR's sparing derives from the superior spatial resolution and inferior transmission through leaves with the former when compared to the latter technique. RA_M120 generally improved OARs sparing compared to IMRT suggesting, given the usage of same MLC, a superior modulation capability (Table 4). The only exception in this pattern is represented by the penile bulb $\left(D_{1} 7.7 v s\right.$. 7.6; Table 4). This OAR is moderately distant from the target and affected by higher scattering, mostly compensated if the High Definition HD_120 MLC is used instead of the Millennium M120.

\section{Discussion}

More than one out of four patients presenting a BF after definitive RT will have clinical evidence of local recurrence within 5 years [18]. Failure to control the prostate is not only a cause of local disease progression but provides possibly a nidus for systemic spread, as shown by the distant metastasis rate in this population [18]. A body of literature predicts however that complications, not limited to but including, the rectum $[19,20]$ and urethra $[21,22]$, after any salvage local therapy in a post-RT setting, is significant. As such, rectal and urethral toxicity is a major concern when using external beam RT as salvage local therapy [23]. We have undertaken a treatment plan comparative study to assess the dose deposition to these OARs, using intensity modulated photons and protons techniques. Overall, IMPT and RA techniques substantially decreased the dose in the intermediate range level to the rectum and urethra (Fig. 3). All the volume and dose metrics for these OARs were substantially decreased with IMPT and RA when compared to IMRT (Table 4). As such, these findings might have bearing on clinical practice for recurrent prostate cancer after RT. RA or IMPT might be an alternative to salvage prostatectomy, cryosurgery or brachytherapy in a selected number of patients.

Non conventional RT, be it IMRT, IMPT or RA, was simulated essentially to capitalize the prerequisite tight dose conformation necessary to administer radiation to these heavily pre-treated prostates. This conformal ability was coupled with the theoretical advantage of hypo fractionation in prostate cancer, while respecting the dose-tolerance of pre-irradiated OARs in the vicinity of the prostate. An increasing body of data now suggests that the $\alpha / \beta$ ratio for prostate is low, possibly in the range of 1-3 Gy [24]. If this metric is accurately low, then hypo fractionated radiation schedules should improve the therapeutic ratio [25]. It was chosen to elect a hypo fractionated radiation schedule for this treatment plan comparison as the dose limiting OARs in vicinity of the GTV was a major issue and may have $\alpha / \beta$ ratios exceeding that for prostate cancer, thus decreasing the probability of toxicity and increasing the probability of cure. Assuming a complete inter-fraction complete repair and no time factor, the total equivalent 

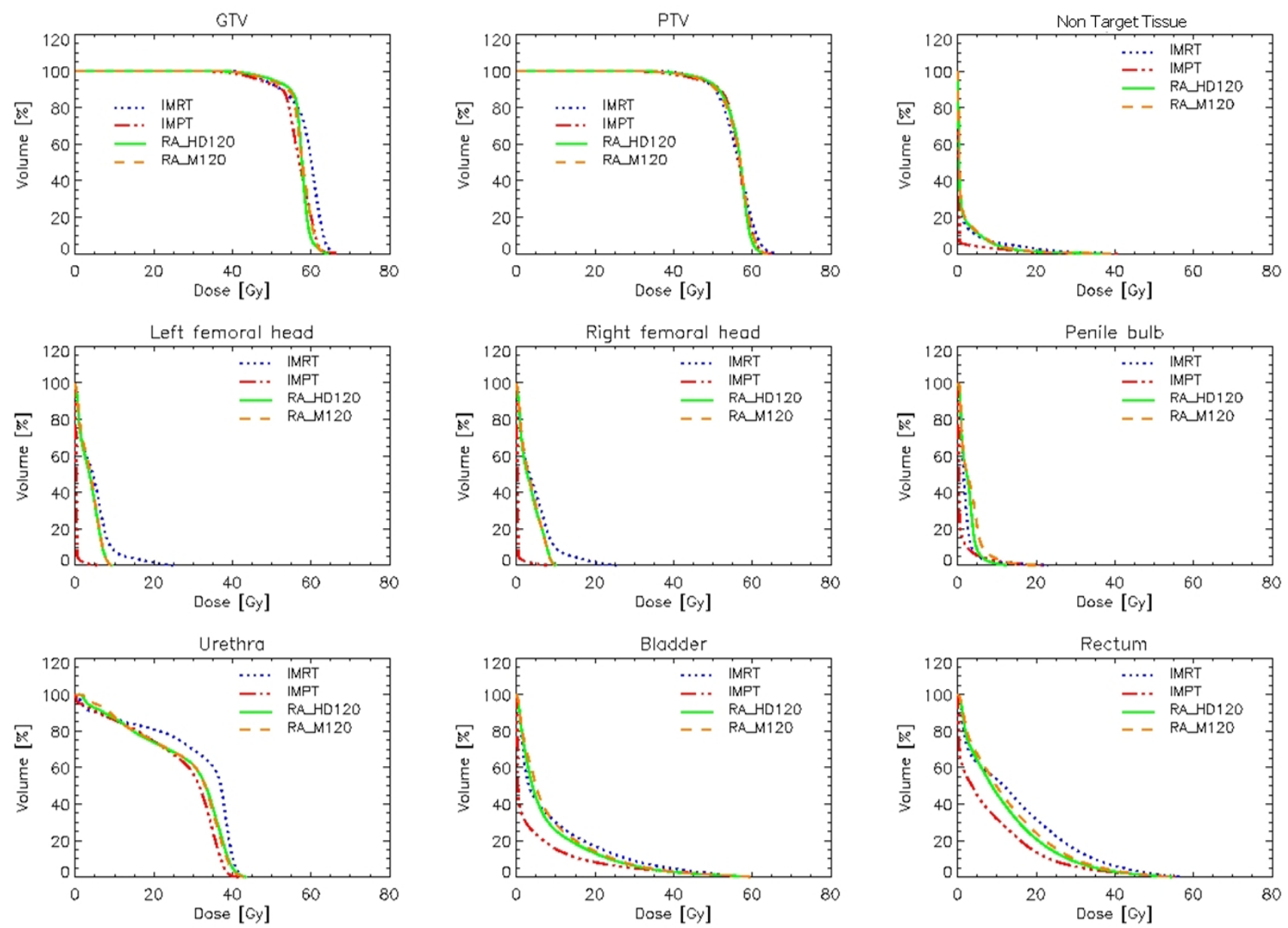

Figure 3

Mean DVHs for CTV, PTV and OARs.

dose of 56 Gy delivered in 14 fractions would be about 88 Gy if the $\alpha / \beta$ ration is 1.5 if delivered at $1.8 \mathrm{~Gy} /$ fraction, according to the presumed $\alpha / \beta$ ratio for prostate cancer using the linear quadratic model.

Biochemical control of prostate cancer patients with recurrent disease may ultimately not be achieved for two main reasons. First, the biochemical failure might be related to the presence of occult metastasis at salvage treatment. It is therefore of paramount importance to appropriately choose patients who are most likely to have local disease only, not limited to but including, interval PSA failure $>3$ years, positive re-biopsy, low Gleason score at re-biopsy, low PSA values at relapse, PET positive intraprostatic tumor, negative bone scan/pelvic imaging studies and PSA-DT $>8$ months. All our patients presented these characteristics for the 6 former factors (1 re-biopsy medically contra-indicated) and all but 1 had a PSA-DT $>$ 8 months [26,27] (Table 1). Second, the local disease may be inadequately addressed by conventional radiology.
Unfortunately, approximately half of all patients will have extraprostatic disease [28] and it is thus critical to optimally define the target volume. It is axiomatic that any suboptimal GTV and PTV delineation may ultimately translate into local failure. For all patients, we have used metabolic imaging in conjunction with endo-rectal MRI. PET imaging with the non-FDG tracers, such as ${ }^{11} \mathrm{C}$ choline, ${ }^{11} \mathrm{C}$-acetate, and ${ }^{18} \mathrm{~F}$-fluorocholine have shown promising results [29]. Notwithstanding the spatial limitation of PET for the staging of prostate cancer (i.e. capsule invasion, $\left.\mathrm{CT}_{3}\right),{ }^{18} \mathrm{~F}$-choline PET has shown an overall sensitivity of $86 \%$ in detecting local recurrent disease in a recent series [30]. Likewise, Reske et al. [31] assessed the value of choline PET/CT for localizing occult relapse of prostate cancer after radical prostatectomy in 49 patients. Focally increased ${ }^{11} \mathrm{C}$-choline uptake in the prostatic fossa was observed in $70 \%$ of patients with histological verification of recurrence. As such, any re-irradiation techniques should deliver radiation to small morphologically and metabolically defined GTV. 
Patient selection for re-irradiation according to clinical and biochemical factors is of critical importance as discussed earlier. First, the physicians have to comprehensively assess the type of failure of her/his recurrent prostate cancer. Second, the site of local failure has to be defined precisely using biopsy and PET CT. Of note, in our small cohort, all patients had a morphological-metabolic and -pathological correlation (Table 2). None less central to treatment success are the tumor geometrical characteristics and localization within the prostate. All our patients presented with small local recurrences, with a mean GTV and PTV of 6.6 and $28.2 \mathrm{~cm}^{3}$, respectively (Table 1). The smaller the tumor, the easier it will be to meet appropriately the OAR's dose constraints for re-irradiation. The 3D locations of these recurrent tumors were however challenging. The urethra was in all but two cases fully surrounded by the GTV. Huang et al. have reported on 47 salvage prostatectomies performed in prostate cancer patients treated with primary RT. Sixty-seven \% of patients had recurrent cancer $\leq 5 \mathrm{~mm}$ from the urethra [28]. This OAR, and not the rectum, was the dose limiting structure in a recent HDR brachytherapy series [23]. This necessitates the application of the most advanced radiation techniques to guarantee satisfactory OAR's conformal avoidance.

All techniques were able to deliver high-dose hypo-fractionated re-irradiation. Cumulatively, IMRT, compared to IMPT or RA, appeared to be less optimal, when certain but not all dosimetric parameters are analyzed (Table 3, 4). The magnitude of the clinical benefit of these latter techniques remains however to be demonstrated. The less favorable IMRT plan comparison metrics results of inferior OAR sparing and of higher target dose heterogeneity and significantly higher GTV and PTV hot spots (Fig. 3).

As expected, IMPT, presented a significantly better sparing of non target tissues but did not offered a substantial improvement of target coverage compared to RA. The usage of the High Definition MLC for RA is somehow advantageous compared to the Millennium MLC for both target and OARs. This fact is noticeable and logical, given the very small size of the GTVs and PTVs. This observed difference between RA_HD120 and RA_M120 may also be clinically not pertinent. RA, with the most generally available Millennium MLC might therefore be considered appropriate also for very small GTVs, offering this modality to a wider number of patients.

Another objective was to assess the capability of the different radiation techniques to manage demanding and opposite planning objectives such as PTV coverage vs. urethra sparing. Such a dosimetric challenge, given the relative position of the two volumes, requires the generation of very steep dose gradients to create in an ideally uniform dose distribution of $56 \mathrm{~Gy}$ a donut hole with a maximum dose of about $67 \%$ (a step of about $20 \mathrm{~Gy}$ in $2-3 \mathrm{~mm}$, i.e. 6-10 Gy/mm). Although all techniques have failed these paradoxical dose-constraints, IMPT and RA techniques could be considered appropriate for these challenging patients (Table 4; Fig. 2). These data are supportive of the sophisticated modulation capabilities of RA with one single arc, despite recent criticisms raised on the basis of over-simplified geometrical assumptions [32].

There were several limitations of our study. First, the small sample size limits the applicability of our conclusions to all prostate cancer patients with recurrent local disease after RT. As only $25 \%$ of these patients could be eligible to local curative treatment [33], clinical judgment (i.e. patient's overall health, morbidity from the local treatment, recurrent tumor characteristics) should always supersede any institutional re-treatment protocols applied indiscriminately to this population. Second, it is axiomatic that any high-dose re-irradiation of the prostate should be undertaken only with appropriate treatment positioning protocols, not limited but including image guidance radiation delivery, robotic couch positioning and prostatic implants for optimal radiation targeting. These issues were purposely not addressed in this dosecomparative study. Third, the localization of the urethra on the planning CT can be problematic, even with the help of an experienced radiologist and CT-MRI fusion. It may be appropriate to catheterize these challenging patients with small catheters during RT simulation. Fourth, only generically dose constraints for OARs were implemented for the RT planning of recurrent prostate cancer in this series. At this juncture, given the potential re-irradiation-induced toxicity, consideration could be given to the prior individual RT plan to adapt each retreatment plans. As such, given the dosimetric metrics of the prior RT, some patients could possibly not be retreated with these techniques. Finally, the issue of delivering radiation with a high dose gradient (i.e. 6 - $10 \mathrm{~Gy} / \mathrm{mm}$ ) to PET defined GTVs has not been addressed in this study. This concern will be developed in a future publication.

\section{Conclusion}

RA, IMPT and IMRT techniques were compared for salvage local treatment in patients with recurrent prostate cancer after RT. All techniques proved to be dosimetrically adequate, with IMPT offering the best sparing of OARs and RA a slightly superior coverage of GTV with an OAR sparing intermediate between IMRT and IMPT. Given limited accessibility of proton facility, RA appears to be a promising treatment solution for particularly small recurrent prostate tumors.

\section{Abbreviations}

RA: volumetric modulated arcs radiation therapy; IMRT: intensity modulated radiation therapy; RT: radiation therapy; IMPT: intensity modulated proton therapy; GTV: 
recurrent gross tumor volume; PET: positron emission tomography; BF: biochemical failure; DVH: dose volume histogram; CI: conformity index.

\section{Competing interests}

LC acts as Scientific Advisor to Varian Medical Systems and is Head of Research and Technological Development to Oncology Institute of Southern Switzerland, IOSI, Bellinzona. Other authors have no conflict of interest.

\section{Authors' contributions}

RM, LC and DCW were responsible for the primary concept and the design of the study; HW, HV, HZ and LC performed the data capture and analysis; LC performed the statistical analysis; DCW and LC drafted the manuscript; DCW and HW reviewed patient data; all authors revised and approved the final manuscript.

\section{Acknowledgements}

This work was supported in part by Grant No. SNSF 3100 A0- I 16547 from the Swiss National Foundation.

\section{References}

I. Kupelian PA, Thakkar VV, Khuntia D, Reddy CA, Klein EA, Mahadevan A: Hypofractionated intensity-modulated radiotherapy (70 Gy at 2.5 Gy per fraction) for localized prostate cancer: long-term outcomes. Int J Radiat Oncol Biol Phys 2005, 63: |463-|468.

2. Kuban DA, Thames HD, Levy LB, Horwitz EM, Kupelian PA, Martinez AA, Michalski JM, Pisansky TM, Sandler HM, Shipley WU, Zelefsky MJ, Zietman AL: Long-term multi-institutional analysis of stage TI-T2 prostat cancer treated with radiotherapy in the PSA era. Int J Radiat Oncol Biol Phys 2003, 57:915-928.

3. Agarwal PK, Sadetsky N, Konety BR, Resnick MI, Carroll PR, Cancer of the Prostate Strategic Urological Research Endeavor (CaPSURE): Treatment failure after primary and salvage therapy for prostate cancer: likelihood, patterns of care, and outcomes. Cancer 2008, I I 2:307.

4. Zagars GK, Pollack A, von Eschenbach AC: Prostate cancer and radiation therapy--the message conveyed by serum prostate-specific antigen. Int J Radiat Oncol Biol Phys 1995, 33:23-35.

5. Langsteger W, Heinisch M, Fogelman I: The role of fluorodeoxyglucose, I8F-dihydroxyphenylalanine, I 8F-choline, and I8Ffluoride in bone imaging with emphasis on prostate and breast. Semin Nucl Med 2006, 36:73-92.

6. Palma D, Vollans E, James K, Nakano S, Moiseenko V, Shaffer R, McKenzie M, Morris J, Otto K: Volumetric modulated arc therapy for delivery of prostate radiotherapy. Comparison with intensity modulated radiotherapy and three-dimensional conformal radiotherapy. Int J Radiat Oncol Biol Phys 2008, 72(4):996-100I.

7. Kjær-Kristoffersen F, Ohlhues L, Medin J, Korreman S: RapidArc volumetric modulated therapy planning for prostate cancer patients. Acta Oncol 2009, 48(2):227-32.

8. Cozzi L, Dinshaw KA, Shrivastava SK, Mahantshetty U, Engineer R, Deshpande DD, Jamema SV, Vanetti E, Clivio A, Nicolini G, Fogliata $A$ : A treatment planning study comparing volumetric arc modulation with RapidArc and fixed field IMRT for cervix uteri radiotherapy. Radiother Oncol 2008, 89: I80-9|.

9. Clivio A, Fogliata A, Franzetti-Pellanda A, Nicolini G, Vanetti E, Wyttenbach $R$, Cozzi L: Volumetric arc modulated radiotherapy for carcinoams of the anal canal. A treatment planning comparison with fixed field IMRT. Radiother Oncol 2009, 92(I): I 18-24.

10. Miralbell R, Vees H, Lozano J, Khan H, Mollà M, Hidalgo A, Linero D, Rouzaud M: Endorectal MRI assessment of local relapse after surgery for prostate cancer: A model to define treatment field guidelines for adjuvant radiotherapy in patients at high risk for local failure. Int J Radiat Oncol Biol Phys 2007, 67:356-36I.

II. Daisne JF, Sibomana M, Bol A, Doumont T, Lonneux M, Grégoire V: Tri-dimensional automatic segmentation of PET volumes based on measured source-to-background ratios: influence of reconstruction algorithms. Radiother Oncol 2003, 69:247-250.

12. Vees H, Senthamizhchelvan S, Miralbell R, Weber DC, Ratib O, Zaidi $\mathrm{H}$ : Assessment of various strategies for I 8F-FET PET-guided delineation of target volumes in high-grade glioma patients. Eur J Nuc Med Mol Imaging 2009, 36: I82-193.

13. Steiner C, Vees H, Zaidi H, Wissmeyer M, Berrebi O, Kossovsky MP, Khan HG, Miralbell R, Ratib O, Buchegger F: Three-phase I8Ffluorocholine PET/CT in the evaluation of prostate cancer recurrence. Nuklearmedizin 2009, 48: I-9. quiz N2-3

14. Casanova N, Zilli T, Rouzaud M, Dipasquale G, Nouet P, Wang H, Escudé L, Mollà M, Linero D, Miralbell R: Sequential dose escalation study with two different hypofractionated IMRT techniques for localized prostate cancer: acute toxicity. Int J Radiat Oncol Biol Phys 2008, 72(I):. S289 (abstract 2268)

15. Ulmer W, Pyyry J, Kaiss W: A 3D photon superposition/convolution algorithm and its foundation on results of Monte Carlo calculations. Phys Med Biol 2005, 50: I 767-90.

16. Chui C, LoSasso T, Spirou S: Dose calculation for photon beams with intensity modulation generated by dynamic jaw or multileaf collimations. Med Phys 1994, 2 I: I 237-I 244.

17. Ulmer $W$ : Theoretical aspects of energy range relations, stopping power and energy straggling of protons. Radiat Phys and Chem 2007, 76: 1089-1107.

18. Lee WR, Hanks GE, Hanlon A: Increasing prostate-specific antigen profile following definitive radiation therapy for localized prostate cancer: clinical observations. J Clin Oncol 1997, I 5:230-238.

19. Donnelly BJ, Saliken JC, Ernst DS, Weber B, Robinson JW, Brasher PM, Rose M, Rewcastle J: Role of transrectal ultrasound guided salvage cryosurgery for recurrent prostate carcinoma after radiotherapy. Prostate Cancer Prostatic Dis 2005, 8:235-242.

20. Nguyen PL, Chen MH, D'Amico AV, Tempany CM, Steele GS, Albert M, Cormack RA, Carr-Locke DL, Bleday R, Suh WW: Magnetic resonance image-guided salvage brachytherapy after radiation in select men who initially presented with favorable-risk prostate cancer: a prospective phase 2 study. Cancer 2007, I I 0: | 485 -| 492

21. Han KR, Cohen JK, Miller RJ, Pantuck AJ, Freitas DG, Cuevas CA, Kim HL, Lugg J, Childs SJ, Shuman B, Jayson MA, Shore ND, Moore Y, Zisman A, Lee JY, Ugarte R, Mynderse LA, Wilson TM, Sweat SD, Zincke $H$, Belldegrun AS: Treatment of organ confined prostate cancer with third generation cryosurgery: preliminary multicenter experience. J Urol 2003, I 70: I | 26- I I30.

22. Sanderson KM, Penson DF, Cai J, Groshen S, Stein JP, Lieskovsky G, Skinner DG: Salvage radical prostatectomy: quality of life outcomes and long-term oncological control of radiorecurrent prostate cancer. J Urol 2006, I 76:2025-203I. discussion 203I2022

23. Lee B, Shinohara K, Weinberg V, Gottschalk AR, Pouliot J, Roach M 3rd, Hsu IC: Feasibility of high-dose-rate brachytherapy salvage for local prostate cancer recurrence after radiotherapy: the University of California-San Francisco experience. Int J Radiat Oncol Biol Phys 2007, 67: I I06-I I I 2.

24. Miles EF, Lee WR: Hypofractionation for prostate cancer: a critical review. Semin Radiat Oncol 2008, I 8:4 I-47.

25. Wong GW, Palazzi-Churas KL, Jarrard DF, Paolone DR, Graf AK, Hedican SP, Wegenke JD, Ritter MA: Salvage hypofractionated radiotherapy for biochemically recurrent prostate cancer after radical prostatectomy. Int J Radiat Oncol Biol Phys 2008 , 70:449-455

26. Zagars GK, Pollack A: Kinetics of serum prostate-specific antigen after external beam radiation for clinically localized prostate cancer. Radiother Oncol 1997, 44:2I3-22I.

27. Zelefsky MJ, Ben-Porat L, Scher HI, Chan HM, Fearn PA, Fuks ZY, Leibel SA, Venkatraman ES: Outcome predictors for the increasing PSA state after definitive external-beam radiotherapy for prostate cancer. J Clin Oncol 2005, 23:826-83I.

28. Huang WC, Kuroiwa K, Serio AM, Bianco FJ Jr, Fine SW, Shayegan B, Scardino PT, Eastham JA: The anatomical and pathological characteristics of irradiated prostate cancers may influence the 
oncological efficacy of salvage ablative therapies. J Urol 2007, 177:1324-1329.

29. Scher B, Seitz M, Albinger W, Tiling R, Scherr M, Becker HC, Souvatzogluou M, Gildehaus FJ, Wester HJ, Dresel S: Value of IICcholine PET and PET/CT in patients with suspected prostate cancer. Eur J Nucl Med Mol Imaging 2007, 34:45-53.

30. Husarik DB, Miralbell R, Dubs M, John H, Giger OT, Gelet A, Cservenyàk T, Hany TF: Evaluation of [( I 8)F]-choline PET/CT for staging and restaging of prostate cancer. Eur J Nucl Med Mol Imaging 2008, 35:253-263.

31. Reske SN, Blumstein NM, Glatting G: [(I I)C]choline PET/CT imaging in occult local relapse of prostate cancer after radical prostatectomy. Eur J Nucl Med Mol Imaging 2008, 35:9-17.

32. Bortfeld T, Webb S: Single-arc IMRT? Phys Med Biol 2009, 54:9-20.

33. Sylvester J, Grimm P, Blasco J, Meier R, Spiegel J, Heaney C, Cavanagh $W$ : The role of androgen ablation in patients with biochemical or local failure after definitive radiation therapy: a survey of practice patterns of urologists and radiation oncologists in the United States. Urology 200I, 58:65-70.

Publish with Bio Med Central and every scientist can read your work free of charge

"BioMed Central will be the most significant development for disseminating the results of biomedical research in our lifetime."

Sir Paul Nurse, Cancer Research UK

Your research papers will be:

- available free of charge to the entire biomedical community

- peer reviewed and published immediately upon acceptance

- cited in PubMed and archived on PubMed Central

- yours - you keep the copyright

Submit your manuscript here:

http://www.biomedcentral.com/info/publishing_adv.asp
BiolMedcentral 